\title{
Preface for the special issue on computational heat transfer
}

\author{
C. Balaji
}

Published online: 21 September 2012

(C) Indian Institute of Technology Madras 2012

The special issue reports the current advancements in the field of computational heat transfer. The area has witnessed rapid strides in the last four decades and the world has seen an explosive growth in the number, range of applicability and robustness of newer computational tools and their increasing application to problems of practical importance in thermal sciences. This issue carries articles that propose better than the state-of-the-art techniques for solving fluid flow and heat transfer problems, those that consider novel variants to existing techniques and demonstrate their use in engineering problems and also those that have employed well known techniques to very complex problems in fields ranging from chemical engineering to nuclear thermal hydraulics.

The first paper in the issue by Yadav et al. reports the results of steady state simulations of a three dimensional model of a super critical $\mathrm{CO}_{2}$ based natural convection loop with end heat exchangers. The study shows that even for a small temperature difference of $18 \mathrm{~K}$, buoyancy is powerful enough to result in high heat transfer rates. Nandakumar et al. in their paper studied the thermal hydraulics of sodium heated once through steam generator of a nuclear reactor by three dimensional simulations and used these results in a one dimensional analysis. The results from the study provide an estimate of a key engineering quantity, namely differential thermal expansion of shells and tubes in the steam generator.

Amit Kumar and Malhotra formulated a two dimensional numerical model of downward flame over a thin solid fuel and solved it to study the effect of a gas phase

C. Balaji $(\bowtie)$

Department of Mechanical Engineering,

Indian Institute of Technology Madras, Chennai, India

e-mail: balaji@iitm.ac.in heat sink (a wire mesh) on the flame spread and flame extinction. The numerical results were validated with experiments and both of them showed that the wire mesh was effective in reducing the flame spread when placed at a distance of $5 \mathrm{~mm}$.

Sengupta et al. carried out a three dimensional conjugate heat transfer analysis of the $\mathrm{BeO}$ reflector assemblies of a $2 \mathrm{~W}$ upgraded Apsara nuclear reactor. The key challenge here is the accomplishment of heat transfer purely by natural convection and the results indicate that the maximum air gap between the aluminium side and $\mathrm{BeO}$ blocks should not be more than $0.3 \mathrm{~mm}$ to keep the temperatures within safe limits.

Biswas et al. considered a new sub-grid model pertaining to large eddy simulation (LES) methodology, namely shear improved Smagorinsky model that can take into account the mean shear arising out of the anisotropy of the flow. The model was tested for the lid driven cavity flow and the predictions were seen to agree well with the experiments as well as the LES model. Kalyana Raman et al. employed the immersed boundary method (IBM) under mixed convection at $\mathrm{Re}=100$ for an elliptic cylinder. The advantage of the IBM was the use of a simple Cartesian grid in the simulations. The critical Richardson number at which shedding gets suppressed was determined for different aspect ratios of the elliptic cylinder. Phongthanapanich and Dechaumpai proposed a new second order accurate characteristic based finite volume element method for analyzing time-dependent scalar convection-diffusionreaction equation in two dimensions. The method combined the finite volume and the finite element methods. It was seen that the method reduces spurious oscillations and does not require an artificial diffusion to improve the solution stability. Song and Tafti evaluated the use of a wall model for the LES of a rotating ribbed duct and compared the 
results with those from a wall resolved LES. They concluded that the wall model captures the gross features of Coriolis forces and predicts the mean heat transfer augmentation/attenuation to within $10 \%$ of the wall resolved LES on the leading and trailing edges of the duct.

Parthasarathy et al. proposed a semi three dimensional porous body model to simulate the primary heat transport of a fast breeder reactor under natural convection. The proposed model was computationally far less expensive. Even so, it was adequate to model and assess the safety of the decay heat removal system under natural convection.

Aurangazaib and Shafie studied the effect of thermal stratification on MHD free convection with heat and mass transfer over an unsteady stretching surface with heat source, Hall current and chemical reaction. They transformed the boundary layer equations and solved them using the Keller box method. They also carried out a detailed parametric study.

The foregoing gives a quick overview of the papers appearing in this issue. The papers are not only on eclectic topics but are consistent with the themes of both the journal and the special issue. We have taken special care to ensure that there is a healthy mix of papers reporting new algorithms or solution techniques and those that have used established techniques to attempt challenging real life problems. Through this issue, we also hope that our readers will get a ring side view of the state-of-the-art and beyond in the fascinating field of computational heat transfer and get motivated to take on even more challenging problems for the benefit of the community and the society at large. 